\title{
Comparative study between diathermy and microdebrider for inferior turbinate reduction surgeries*
}

\author{
Shrinivas S. Chavan', Abhishek D. Khond', Pallavi Saple², Vinayak R. Kurle \\ 'Department of Otorhinolaryngology, Grant Government Medical College and Sir J.J. group of hospitals, Mumbai, India \\ 2Dean, Grant Government Medical College and Sir J.J. group of hospitals, Mumbai, India
}

Rhinology Online, Vol 3: 1 - 7, 2020

http://doi.org/10.4193/RHINOL/19.033

*Received for publication:

October 21, 2019

Accepted: January 29, 2020

Published: February 17, 2020 (1) 
50-60 mm in length, $7.5 \mathrm{~mm}$ in height, and $3.8 \mathrm{~mm}$ width $^{(3)}$. The inferior turbinate has two important functions, i.e. resistor function and diffusor function. Resistor function is carried out by contributing in inspiratory resistance. Higher the resistance, higher the negative intrathoracic pressure required for inspiration, which in turn increases pulmonary ventilation and venous backflow to lungs and heart ${ }^{(4)}$. Inferior turbinate also obstructs nasal valve area, which changes the inspiratory lamellar airstream into turbulent flow. More the turbulence, more is the interaction between air and nasal mucosa, which enhances the humidification, warming up, and cleansing of air. Thus it plays a critical role in this process because of large mucosal surface and extensive blood supply. This is 'diffusor function' of inferior turbinate ${ }^{(4)}$. They also help in nasal defense system (mucociliary transport, humoral and cellular defense). All of these functions require a large amount of normally functioning mucosa, submucosa, and turbinate parenchyma ${ }^{(4)}$.

Nasal obstruction due to inferior turbinate can be explained by three anatomic variations (soft tissue, bony and mixed). Soft tissue hypertrophy is very common and is seen in cases of chronic rhinitis like allergic rhinitis, nonallergic rhinitis with eosinophilic syndrome, or iatrogenic rhinopathy. Bony hypertrophy is caused by prominent inferolateral turn and progressive ossification of bone $^{(5)}$. Mixed type is also seen in chronic rhinitis cases ${ }^{(5)}$. Although most cases of ITH can be managed medically by antihistaminic, topical decongestants, corticosteroids but surgical intervention sometimes becomes necessary in certain nonresponding patients which are managed by Submucous Inferior Turbinate Reduction (ITR) surgery ${ }^{(6,7)}$. Basic principle of these ITR surgeries is to reduce the turbinate size to decrease symptoms of nasal blockage but they differ from each other based on preservation of normal physiological function ${ }^{(6)}$.

In past, total or partial turbinectomy were popular, which were short surgical procedures and easier to perform and did not require sophisticated instruments or skills, but these procedures did not maintain physiological function and were associated with significant blood loss and left the cut surface prone to crust formation and synechiae ${ }^{(6,8)}$.

To prevent these complications, many other techniques were introduced for ITR which included submucous reduction of inferior turbinate using diathermy, cryotherapy, laser therapy, microdebrider and radiofrequency ablation ${ }^{(7)}$.

Large variation in surgical techniques available denotes lack of consensus on optimal technique ${ }^{(7)}$. Although submucosal diathermy still remains a very popular technique because of technical ease and lack of complication. But with the advent of microdebrider to rhinosurgery by Setliff et al, many surgeons have recently started using microdebrider for the same ${ }^{(7)}$. Thus, this study was conducted with the objective to compare the outcome following Inferior turbinate reduction using microdebrider and diathermy.

\section{Materials and Methods}

\section{Study design}

This was a prospective interventional and comparative study conducted between October 2017 to March 2019 in Department of Otorhinolaryngology, Sir J.J. Hospital attached to Grant Government Medical College, Mumbai, India. A total of 150 patients were enrolled in this study, after taking an informed consent and acquiring the ethical committee clearance. Patients attending ENT OPD with persistent nasal blockade, sneezing, post nasal drip and persistent nasal discharge were screened and subjected to anterior rhinoscopy and nasal endoscopy.

\section{Inclusion criteria}

Patients with ITH due to any cause like persistent allergic rhinitis not relieved by medications, nonallergic rhinitis with eosinophilic syndrome, or iatrogenic rhinopathy were included in this study.

\section{Exclusion criteria}

Individuals with any other causes of nasal blockade other than ITH, patients with proven granulomatous infection of nose and patients who have previously underwent nasal surgery were excluded from the study. Enrolled patients were subjected to pre-operative assessment using Nasal obstruction symptoms evaluation scale ${ }^{(9)}$ (NOSE score - Table 1), endoscopic findings, and mucociliary transit time (MTT) using saccharin test.

\section{Nasal endoscopy}

Endoscopic findings in all patients were noted to rule out any other endonasal pathology other than ITH and nasal patency was assessed at level of internal nasal valve area in terms of percentage.

\section{Mucociliary transit time (saccharin test)}

With the patient in seated position, a saccharin granule was placed on the anterior part of inferior turbinate, and time required to experience a sweet taste was determined. Then patient was asked to swallow every 30 seconds; immediately after the patients tasted the saccharin, the test was stopped. Time was measured in minutes. All patients underwent a screening Computed Tomography of Paranasal sinuses (CT PNS) scan to rule out co-existing nasal pathology. Patients were randomized into two pools (Pool A and Pool B) by chit allocation technique. Each pool consists of 75 patients. Patients in pool A underwent Submucous ITR using Diathermy (SITRD) in pool B underwent Submucous ITR using Microdebrider (SITRM).

All patients were operated by same surgeon who was blinded with respect to study designs and study details. All patients in pool A and pool B were followed up at 7th, 15th, 30th, 45th, and 60 th postoperative day with same postoperative care. NOSE scores and nasal Endoscopy was performed on the aforementi- 
Table 1. Nose score (nasal obstruction symptoms evaluation scale) ${ }^{(8)}$ using a questionnare.

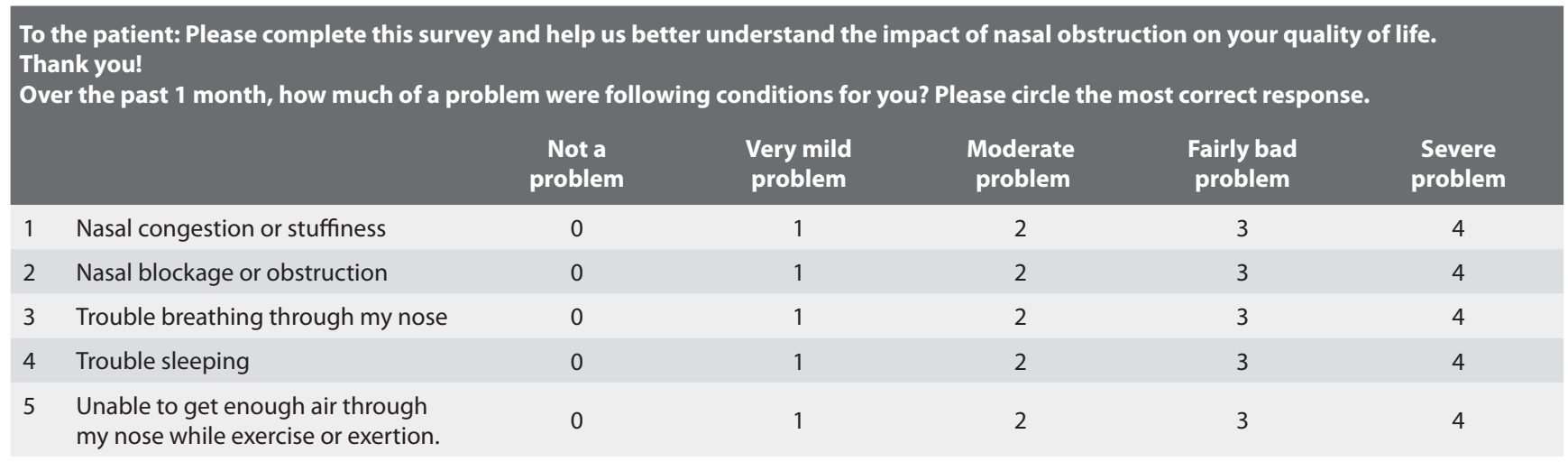

oned follow-up days. MTT was performed at the end of 60th day. Above three parameters were compared pre-operatively and post-operatively between pool A and pool B. Possible complications arising from both techniques like postoperative crusting and synechiae formation were also documented on aforementioned days.

\section{Procedure}

All procedures were performed under general anaesthesia. Patient in supine position was painted and drapped. Zero degree endoscope introduced and size of inferior turbinate assessed. Inferior turbinate infiltrated with $2 \%$ Lignocaine + Adrenaline. In pool A, 7.5cms, 22 gauge spinal needle was used as monopolar probe and inserted longitudinally into the anterior end of inferior turbinate inferior surface. SITRD was done at number 40 power setting. The cautery was done till the anterior end of turbinate blanches avoiding charring. Similar procedure was repeated on medial and superior surface then posterior part of the turbinate.

In pool B, longitudinal incision was taken over the anterior aspect of inferior turbinate of about $0.5 \mathrm{~cm}$ using no. 15 sterile surgical blade and a $4 \mathrm{~mm}$ Microdebrider straight blade inserted into the inferior turbinate longitudinally through the incision site. Submucosal debridement of inferior turbinate along with shaving off a part of bony turbinate was done keeping the mucosa intact along the entire length of inferior turbinate upto choana.

In both pool A and B, at the end of procedures, nasal cavity was packed using antiseptic ointment and liquid paraffin-soaked roller gauze. Nasal pack was removed after 48 hours and Postoperative nasal douching given for 15 days. Follow up was done on the 7th,15th, 30th, 45th and 60th day.

\section{Data analysis and statistical tests}

All the collected data was entered in Microsoft Excel sheet. It was then transferred to SPSS ver. 17 software for statistical analysis. Quantitative data was presented as mean and standard deviation and comparison of the two study groups was done using unpaired t-Test. Pre-operative and post -operative quantitative data of each surgical technique was compared using paired t-Test. Qualitative data was presented as frequency and percentage and analysed using chi-square test. A p-value of $<0.05$ was considered as statistically significant.

\section{Results}

In this study 150 patients were assessed with total study duration of 18 months. In pool A, 75 patients were operated of which 57 were males and 18 were females. In pool B, 75 patients were operated of which 48 were males and 27 were females.

Overall, the maximum incidence was found in 3rd decade (34\%), with minimum age of patient being 13 years and maximum being 54 years. Mean age in SITRD was 29.48 years and in SITRM was 28.28 years (Figure 1).

\section{NOSE score}

The mean pre-operative NOSE score in pool A was 76 whereas in pool B was 84 .

During post-operative follow up, mean NOSE score in pool A on day 7 th, 15 th, 30th, 45th and 60th were 30.65, 29.3, 31.3, 32.65 and 32.65 , respectively, and in pool B on aforementioned days were $28,24.65,21,16.65$ and 14, respectively (Figure 2 ).

In both the techniques, when pre-operative and post-operative (day 60) NOSE scores were compared using paired T test, there was definite improvement in NOSE score post-operatively and the result of the test was statistically highly significant with $p$-value $<0.01$. On comparing the NOSE score using unpaired $T$ test, between the two techniques on each follow up days respectively, it was found that SITRM provided better improvement than SITRD on each day and the results were highly significant with $\mathrm{p}$-value $<0.01$.

\section{Nasal endoscopic findings}

During nasal endoscopy of each patient on pre-operative and post-operative follow up days, percentage thickness of inferior 


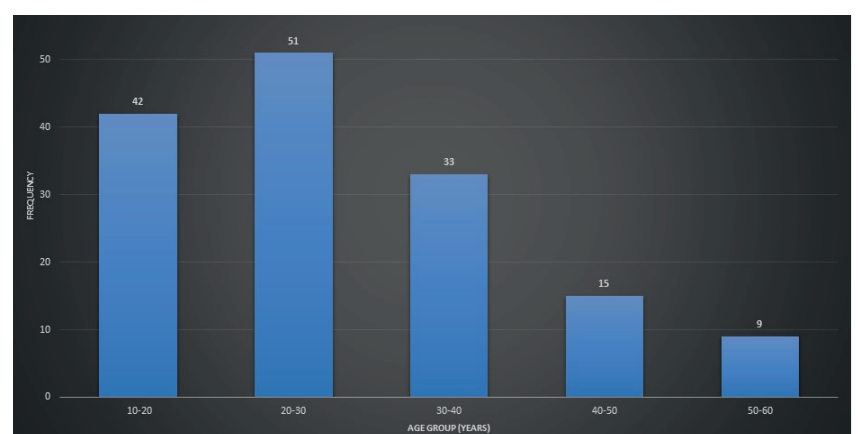

Figure 1. Age-wise distribution of study population.

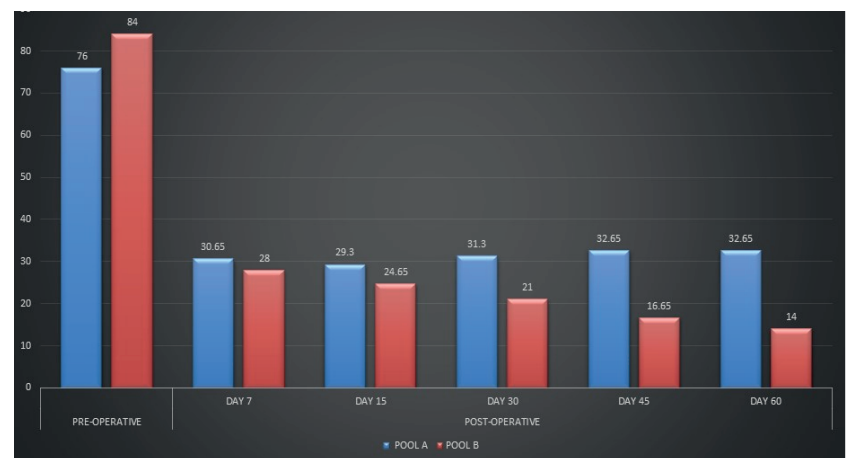

Figure 2. Mean nose core (diathermy vs microdebrider).

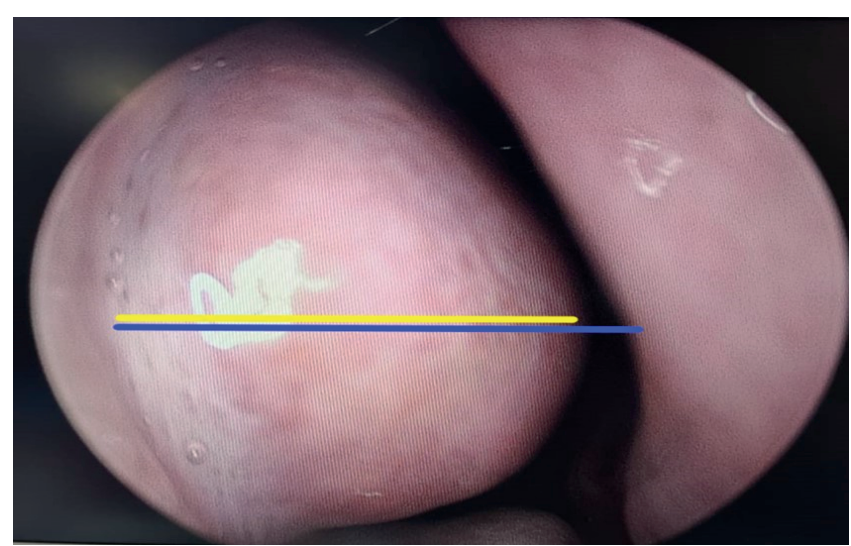

Figure 3. Percentage thickness of inferior turbinate(length of $y$ llow lin in $\mathrm{mm}$ ) as compar $\mathbf{d}$ to maximum width of inferior nasal valve area(length of blue line in $\mathrm{mm}$ ) in Right nostril of pre-operative patient.

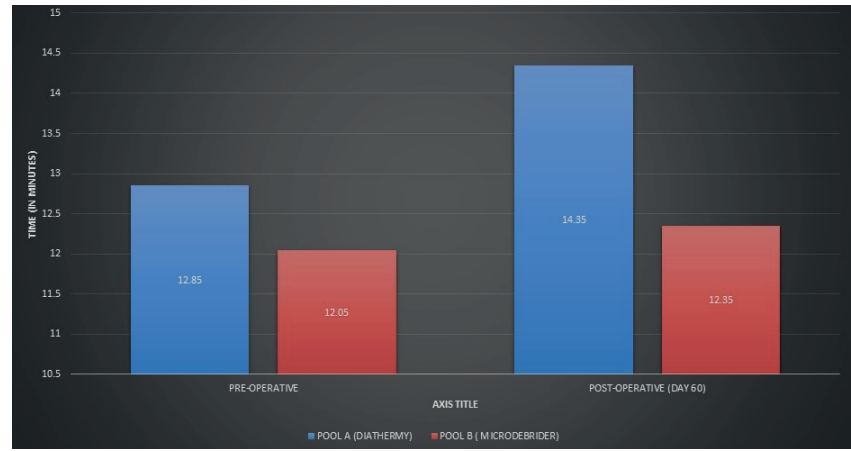

Figure 4. Mean nasal mucociliary transit time (in minutes). turbinate was calculated at the level of internal nasal valve area. The maximum width of internal nasal valve area was considered to be $100 \%$ and percentage was calculated of width of valve area covered by inferior turbinate in each case for both nostrils separately (Figure 3).

The pre-operative mean inferior turbinate size (\%) in pool A in right and left nostrils was $73.66 \%$ and $74.66 \%$ respectively. The pre-operative mean inferior turbinate size (\%) in pool B in right and left nostril was $82.66 \%$ and $84.66 \%$, respectively. When comparison was made between pre-operative and post-operative (day 60) inferior turbinate size (in \%), it was noted that there was significant reduction in size of inferior turbinate in both techniques in both nostrils, with mean day 60 inferior turbinate size being $45.66 \%$ (right side) and $45.66 \%$ (left side) in SITRD. In SITRM, day 60 values were $32.33 \%$ (right side) and $32.66 \%$ (left side). This data was compared using paired $\mathrm{T}$ test and difference in inferior turbinate size between pre-operative and post-operative (day 60 ) was found to be statistically significant with $p$ value of $<0.01$ in both nostrils using both the techniques (Table 2).

On comparing the percentage size of inferior turbinate using unpaired T test, between the two techniques on each follow up days in both nostrils separately, it was found that both pool A and $B$ gave comparable results ( $p$ value $>0.05$ ) on post-operative day 7 with no technique being statistically better than other. But on further follow up days, the results showed SITRM provided better reduction in the size of inferior turbinate as compared to SITRD on each follow up days and the results were highly significant with $p$-value $<0.01$.

\section{Saccharin test}

The mean MTT in pre-operative and post-operative (day 60) cases was 12.85 minutes and 14.35 minutes in pool $A$, whereas 12.05 minutes and 12.35 minutes in pool B (Figure 4). When preoperative and post-operative (day 60) MTT was compared using paired $T$ test, there was significant increase in transit time in case of SITRD ( $p$-value $<0.05$ ). But there was no significant prolonging $(p=0.14)$ of nasal MTT in SITRM, when compared pre and postoperatively.

\section{Post-operative evaluation}

Along with NOSE score, nasal endoscopy and Saccharin test, patients were also followed up for post -operative complications like crusting and synechiae. Minimal bleeding (few drops) was seen in few patients post-operatively after nasal pack removal which stopped spontaneously after few minutes. There was no significant difference between the two groups in terms of nasal bleeding.

Crusting

Presence or absence of nasal crusting was noted in all patients during post-operative follow-up days. Overall 65/75 patients in 
Table 2. Mean of inferior turbinate size (in \% of internal nasal valve area).

\begin{tabular}{|c|c|c|c|c|c|c|c|}
\hline & \multirow[t]{2}{*}{ Side } & \multirow[t]{2}{*}{ Pre-operative } & \multicolumn{5}{|c|}{ Post -operative follow up } \\
\hline & & & Day 7 & Day 15 & Day 30 & Day 45 & Day 60 \\
\hline \multirow{2}{*}{ Pool A } & Right & 73.66 & 40.33 & 40.66 & 41.66 & 43 & 45.66 \\
\hline & Left & 74.66 & 39.33 & 40.66 & 40.33 & 41.66 & 45.66 \\
\hline \multirow{2}{*}{ Pool B } & Right & 82.66 & 39 & 38 & 35 & 32.66 & 32.33 \\
\hline & Left & 84.66 & 38.33 & 36.66 & 35.66 & 34 & 32.66 \\
\hline
\end{tabular}

SITRD and 60/75 patients in SITRM presented with nasal crusting on day 7 follow-up. There was significant reduction in number of cases showing nasal crusting in further follow-up days with almost all the cases free of crusting in both techniques on day 60 . Synechiae formation

Post-operative synechiae formation is seen in both techniques in early post-operative period and required follow-up minor procedure like synechiae release. In SITRD cases, $33.33 \%$ had synechiae formation on post-operative day 7 in comparison to $26.66 \%$ in SITRM. On further follow up, negligible cases showed synechiae formation on day 15 , with no evidence of synechiae on day 30,45 and 60 in both techniques.

\section{Discussion}

ITH is one of the most common cause of nasal blockage for patients to seek an otorhinolaryngologist, which is often seen in cases of allergic rhinitis, nonallergic rhinitis with eosinophilic syndrome, or iatrogenic rhinopathy ${ }^{(6,10)}$. ITH usually is due to swelling of submucosa caused by dilatation of venous sinusoids. Significant cases respond to antihistamines or local decongestant, however; occasionally ITH is due to submucous fibrosis rendering the turbinate incapable of decongestion and hence need surgery ${ }^{(4)}$.

A variety of surgical procedures are performed for managing $\mathrm{ITH}$, but there is no completely effective therapy. The main aim of turbinate surgery has to be preservation of well-functioning mucosa, along with creation of sufficiently large air space to ensure the humidification and purification of air and maintenance of a physiological airway resistance ${ }^{(11)}$. Any method should be judged by two basic criteria: efficacy of the technique in reducing nasal obstruction and its ability to preserve the nasal mucosa ${ }^{(4)}$. Hol and Huizing who evaluated 13 surgical techniques that have been used for ITH over 130 years and concluded that intraturbinal turbinate reduction should be the method of choice $^{(4)}$. In the mid-1990s, the advent of powered instrumentation like microdebrider greatly helped surgeons to treat ITH in a better way ${ }^{(8)}$.

In this study, males (105) outnumbered females (45) with male: female ratio of 2.3:1. Our study was comparable to other studies carried out by Hassoun et al. in Iraq, which reported a male : female ratio of $1.7: 1^{(6)}$. It can be postulated that more occupational exposure to pollutants in Indian setting, increases the chances of allergic rhinitis induced ITH in males. The present study also showed higher incidence of ITH in middle aged working population with peak incidence in 3rd decade (34\%) of life. This data correlates well with studies done by Hassoun et al. ${ }^{(6)}$ and possible explanation for higher trend in middle aged group can be due to more occupational exposure to allergen in middle aged working population.

In general, both techniques were well tolerated by the patients. The major differences between SITRM and SITRD were found in terms of nasal patency with respect to reduction in size of inferior turbinate, NOSE score, mucociliary transit time, post-operative synechiae, and crusting. Although, the measuring of the width of air space in the nasal cavity is not a standardized test, in this study found that the results of this technique correlate well with the patients' subjective sensation of nasal blockage. This was also confirmed in study by Friedman et al., which showed that the visual identification of turbinate reduction combined with the elimination of symptoms speaks for effective turbinate reduction $^{(12)}$.

The improvement in nasal patency was studied in form of NOSE score and reduction in inferior turbinate size. Relief of nasal blockage was more pronounced and faster and statistically more significant after SITRM than after SITRD, because inflammatory oedema is more severe and last longer time after diathermy, in addition, diathermy involve no volumetric reduction, i.e. the technique depends on post-operative healing and fibrosis which cannot be predicted with confidence. Joniau et al. performed their study on 19 patients, they did powered turbinoplasty on one side and submucosal cauterization on the other, and found that powered turbinoplasty was superior to submucosal cauterization in all aspects of the assessment ${ }^{(13)}$. A significant difference $(p<0.05)$ was noted for postoperative crusting, endoscopic scoring of turbinate size, and mean area at the level of the nasal valve. In addition, the results of powered turbinoplasty were still apparent on long term follow-up, whereas submucosal cauterization was associated with recurrence. Similar results were obtained by Mahlon et al. ${ }^{(14)}$ who performed microdebrider assisted turbinoplasty in 100 patients and 
achieved postoperative improvement in nasal patency in 93\% of the patients, whereas improvement in nasal obstruction was seen in $91 \%$ cases in study done by Lee and Chen ${ }^{(15)}$. Improvement of nasal symptoms were seen as early as within 2 months after microdebrider assisted turbinoplasty which were supported by other studies like Friedmann et al. and Hegazy et al. ${ }^{(12,16)}$. The results of our study demonstrated significant improvements of NOSE scores $(p<0.001)$ and reduction of inferior turbinate size at 60 th day during the postoperative period by using both microdebrider and diathermy with these outcomes comparable to other previous studies on the outcomes of each equipments and demonstrated promising results of both these treatments. Microdebrider produced excellent reduction in the size and provided symptomatic relief at 7th, 15th, 30th day and which persisted till 60th day. SITRD, though it produced comparable results with SITRM, at 60th day was not able to prevent the symptoms from recurring. It has to be considered that neither the symptoms nor the size of the turbinate in this group has increased to the preoperative size in the group who underwent monopolar diathermy. Still the results were statistically variable when compared to the other group at the same point of time. This indicate the requirement of repeating the procedure in at least a few cases. The possible explanation of this could be the fact that microdebrider shaves off both turbinate bone and soft tissue whereas diathermy causes just submucosal soft tissue fibrosis. Reduction of the bone creates more space, whereas surgery on submucosal tissue creates scarring that minimizes the engorgement of the inferior turbinates of patients with rhinitis ${ }^{(17)}$.

Ciliary function which determines the MTT forms an important defence mechanism that protects the respiratory system. As also shown in other previous studies ${ }^{(18-20)}$, saccharine transit time showed a significant impairment in patients where diathermy was used because of thermal mucosal damage caused in diathermy as compared to microdebrider. Preservation of mucosa also improves the chances for continued function of the inferior turbinates to warm and humidify the inspired air ${ }^{(12)}$.

In terms of post-operative surgical complications, SITRD produced significantly more crusting and synechiae formation as compared to SITRM in early post-operative period till 30th day and required surgical intervention like synechiae release procedure and suction clearance of crustings. However, during 45th day and 60th day follow ups there was no difference noted in the two techniques in terms of crusting and synechiae formation, with nasal mucosa healthy in almost all cases operated by both diathermy and microdebrider.

In some cases of SITRD (about 3\%), mucosal changes similar to atrophic rhinitis were noted which may be related to excessive cauterization of nasal mucosa leading to roomy nasal cavity due to over shrinkage of inferior turbinate following fibrosis. But these changes were only evident during 60th day follow up and further follow up should be done in terms of these aspect to look for chronic changes in nasal mucosa in operated cases of inferior turbinate reduction by both diathermy and microdebrider.

The main disadvantage in SITRM is the high cost for the procedure as microdebrider blade is for single use only, hence less affordable. Monopolar cautery using spinal needle can be done in any surgical setting which makes it cost effective. Microdebrider is also technically more demanding and requires surgical skills as compared to diathermy. Consequently, microdebrider has an edge over diathermy in attaining both symptomatic relief and lesser post-operative complications over a long period. SITRD, though it provides comparable reduction postoperatively, worsening of symptoms and signs were noticed occasionally.

\section{Conclusions}

To conclude submucosal resection with microdebrider produce better results in the treatment of inferior turbinate hypertrophy, both in the short term and long term compared to the submucosal diathermy, where the latter produce comparable results in the early postoperative period. Mucociliary function is better preserved with microdebrider. But cost effectiveness of diathermy outweighs benefits of microdebrider at present in Indian scenario. Limitation of this study was that different etiological causes for inferior turbinate reduction were not taken into consideration and ITH due to any cause were included in the study irrespective of its cause. Another limitation of this study was that objective method of nasal patency assessment like rhinomanometry were not used due to cost restrains. A more elaborate larger randomized studies with use of rhinomanometry would definitely be helpful to confirm or refute the same.

\section{Acknowledgement}

Not applicable.

\section{Authorship contribution}

All authors have read and approved the final manuscript. AK and VK were responsible for investigating and evaluating cases as per inclusion and exclusion criteria. All the cases were operated by SSC. Final drafting of the article was done by AK and VK under guidance of SSC. Entire research work was co-ordinated and supervised by PS.

\section{Conflict of interest}

The authors have no conflicts of interest to declare.

\section{Ethics approval and consent to participate} Not applicable.

\section{Consent for publication}

Not applicable. 


\section{Availability of data and materials} Not applicable.

\section{Funding}

Not applicable.

\section{References}

1. Hsu DW, Suh JD. Anatomy and physiology of nasal obstruction. Otolaryngologic Clin N Am. 2018;51(5):853-65.

2. Hesham A, Badran H, Hussein A, Amin S Salah M. Intraturbinal versus extraturbinal microdebrider-assisted inferior turbinoplasty: Preliminary results. Egyp J Ear, Nose, Throat Allied Sci. 2013: 15. 10.1016/j.ejenta.2013.09.004.

3. Lund VJ, Stammberger H, Fokkens WJ, et al. European position paper on the anatomical terminology of the internal nose and paranasal sinuses. Rhinology. Supplement. 2014;24:1-34

4. Hol MK, Huizing EH. Treatment of inferior turbinate pathology: a review and critical evaluation of the different techniques. Rhinology. 2000;38(4):157-66

5. Berger G, Balum-Azim M, Ophir D. The normal inferior turbinate: histomorphometric analysis and clinical implications. Laryngoscope. 2003;113(7):1192-8.

6. Hassoun YL. Comparison and evaluation effect between microdebrider assisted turbinopalsty and submucosal diathermy of the inferior tubinates techniques. Am J Res Comm, 2015, 3(7): 47- 56

7. Ragab A, Elbanhawy O, Khashba A, Ali A AbdelAziz M. Microdebrider-assisted turbinoplasty against submucosal cauterization in inferior turbinate hypertrophy. Menoufia Med J. 2016;29(3):504

8. Hackman TG, Ferguson BJ. Powered instrumentation and tissue effects in the nose and paranasal sinuses. Curr Opin Otolaryngol Head Neck Surg, 2005; 13, 1 : 22-26.

9. Stewart MG, Witsell $D L$, Smith $T L$, Weaver EM, Yueh B, Hannley MT.
Development and validation of the Nasal Obstruction Symptom Evaluation (NOSE) scale. Otolaryngol. Head Neck Surg. 2004;130(2):157-63

10. Lorenz K, Maier, H. [Microdebrider-assisted inferior turbinoplasty: Minimally invasive technique for the treatment of nasal airway obstruction caused by enlarged turbinates.]. HNO Lorenz. 2012.

11. Scheithauer MO. Surgery of the turbinates and "empty nose" syndrome GMS Curr Top Otorhinolaryngol Head Neck Surg. 2010, 9, Doc03.

12. Friedman M, Tanyeri H, Lim J, Landsberg R, Caldarelli, D. A safe, alternative technique for inferior turbinate reduction. Laryngoscope 1999; 109(11):1834-1837.

13. Joniau S, Wong I, Rajapaksa S, Carney SA, Wormald PJ. Long-term comparison between submucosal cauterization and powered reduction of the inferior turbinates. Laryngoscope. 2006;116(9):1612-6.

14. Van Delden MR, Cook PR, Davis WE. Endoscopic partial inferior turbinoplasty. Otolaryngol Head Neck Surg.1999;121(4):406-9.

15. Lee CF, Chen TA. Power microdebriderassisted modification of endoscopic inferior turbinoplasty: a preliminary report. Chang Gung Med J. 2004;27(5):359-65.

16. Hegazy HM, El Badawy MR, Hassan AA. Endoscopic Submucous Inferior Turbinate Reduction with Microdebrider - A Study of 50 Case. Tanta Med Sci J, 2007; 2,1, 194-199.

17. Zeynep K, Kursat C. Comparison of radiofrequency tissue volume reduction and submucosal resection with microdebrider in inferior turbinate hypertrophy. Otolaryngol Head Neck Surg. 2008; 138, 176-181.

18. Kesornsukhon N. Radiofrequency volumetic tissue reduction for treatment of inferior turbinate hypertrophy. Region 6-7 Med J. 2007; 26(2):197-204

19. Kesornsukhon N. Comparison of RFTVR and SMRM in treatment of inferior turbinate hypertrophy. Chachoengsao Hospital J. 2008; 96:97-104

20. Kesornsukhon N. Comparison of outcome of treatment for inferior turbinate hypertrophy: RFTVR and SMRM. Bull Dept Med Serv. 2009; 34:81-7.

21. De Corso E, Bastanza G, Di Donfrancesco $V$, et al. Radiofrequency volumetric inferior turbinate reduction: long-term clinical results. Acta Otorhinolaryngologica Italica. 2016:36(3):199.

22. Sommer F, Scheithauer MO, Hoffmann TK, Grossi AS, Hauck K, Lindemann J. Value of turbinoplasty in rhinosurgery-a controlled randomized study. Rhinology. 2019;57(5):352-7.

\section{Dr. Vinayak Kurle}

Department of Otorhinolaryngology

Grant Government Medical College

and

Sir J.J. group of hospitals

Mumbai

India

Mobile: 9481164734

E-mail: vinukurls911@gmail.com

ISSN: 2589-5613 / @2020 The Author(s). This work is licensed under a Creative Commons Attribution 4.0 International License. The images or other third party material in this article are included in the article's Creative Commons license, unless indicated otherwise in the credit line; if the material is not included under the Creative Commons license, users will need to obtain permission from the license holder to reproduce the material. To view a copy of this license, visit http://creativecommons.org/licenses/by/4.0/ 